\title{
Polimorfismo -174 G/C del gen promotor de interleuquina 6 en mujeres con diabetes mellitus tipo 1
}

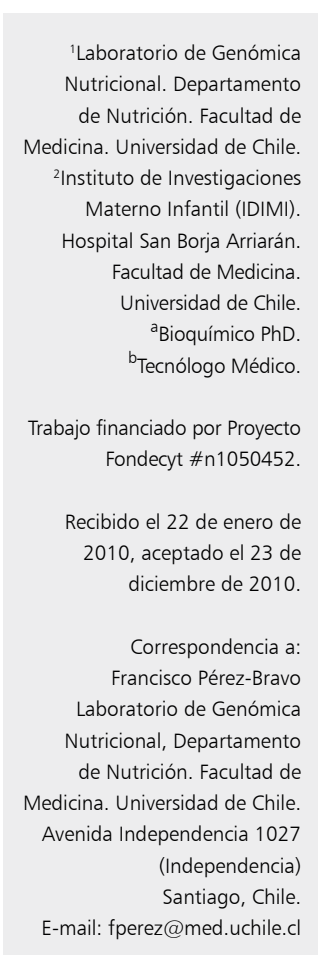

\author{
FRANCISCO PÉREZ-BRAVO ${ }^{1, a}$, FRANCISCA SOTO M. ${ }^{1, b}$, \\ PATRICIA LÓPEZ A. ${ }^{2, b}$, FRANCISCA EYZAGUIRRE C. ${ }^{2}$, ETHEL CODNER ${ }^{2}$
}

\section{-174 G/C polymorphism of interleukin 6 gene in women with type 1 diabetes}

Background: A polymorphism located in the promoter region (-174 G / C) of interleukin 6 (IL-6) has been linked to early onset of type 1 diabetes (T1D) and increased body mass index (BMI). Aim: To evaluate the possible association of this -IL-6 gene 174 G / C polymorphism with T1D, BMI and metabolic control in T1D patients in a case-control study. Patients and Methods:-174 G / C polymorphisms were analyzed by polymerase chain reaction and restriction fragment length polymorphism in 145 women with T1D and 103 healthy controls. BMI and BMI-Z-scores were tabulated and metabolic control was recorded. Results: There was a higher frequency for the $C$ allele in T1D patients compared with the control group (21\% versus $14.1 \%, p=0.047$ ). No significant differences in genotype frequencies for the -174 G/C polymorphism of IL-6 gene between patients with T1D and controls, were observed. There were no significant associations with T1D and BMI. Conclusions: $A$ higher frequency of $C$ allele in women with T1D was the only finding in this series, suggesting that the polymorphic variant is not related to weight gain in these patients.

(Rev Med Chile 2011; 139: 158-164).

Key words: Body mass index; Diabetes mellitus, type 1; Interleukin-6

\section{L} a diabetes mellitus tipo 1 (DM1) es una enfermedad compleja caracterizada por la destrucción selectiva y progresiva de las células $\beta$ pancreáticas productoras de insulina y cuya incidencia está aumentando progresivamente en todo el mundo ${ }^{1}$.

Diversos factores, tanto genéticos (numerosos genes involucrados en la respuesta inmune), como ambientales (virus, compuestos químicos, dieta y estrés), han sido propuestos como los principales participantes en este fenómeno ${ }^{2-4}$.

La resistencia insulínica y la inflamación corresponden a dos fenómenos que tradicionalmente se han relacionado a la diabetes mellitus tipo 2 (DM2), sin embargo, la evidencia de la presencia de estos mecanismos en la DM1 es cada día más fuerte ${ }^{5,6}$. La resistencia insulínica es un fenómeno frecuente en la DM1, especialmente durante la etapa puberal. En mujeres con DM1, este fenómeno podría ser muy relevante en la patogenia del síndrome de ovario poliquístico, ya que junto a la hiperinsulinemia, podrían explicar el aumento de los andrógenos en estas pacientes ${ }^{7}$.

La interleuquina 6 (IL-6) es una citoquina multifuncional producida por diferentes tipos celulares, incluyendo células del sistema inmune (células $B$, células $T$ y macrófagos), células dendríticas, fibroblastos, células endoteliales, miocitos, tejido adiposo, y células $\beta$ pancreáticas. Esta citoquina media en la respuesta inflamatoria y de estrés. Se ha calculado que la tercera parte de la concentración circulante de IL-6 proviene del tejido adiposo ${ }^{8}$. De acuerdo con observaciones recientes, la concentración circulante de IL-6 se 
relaciona con la acción de la insulina ${ }^{9-12}$, como factor de riesgo cardiovascular y con el desarrollo de obesidad. Concentraciones elevadas de IL-6 se han asociado con la DM2, con elevado índice de masa corporal, dislipemias y con hipertensión arterial ${ }^{12}$.

Diversos polimorfismos en la región promotora del gen IL-6 han sido descritos ${ }^{13}$. Uno de ellos, el polimorfismo $-174 \mathrm{G} / \mathrm{C}$, afecta la transcripción del gen de IL-6, con una mayor producción de IL-6 ${ }^{14}$. Jahromi y cols, el año $2000^{15}$, sugieren que este polimorfismo estaría asociado con una mayor susceptibilidad al desarrollo de DM1, describiéndose al genotipo homocigoto G/G como altamente asociado a la enfermedad. Durante el año 2005, Gillespie y cols ${ }^{16}$, demuestran que este polimorfismo se asociaba con la edad de debut en mujeres con DM1, efecto no observado en hombres. Estos resultados podrían relacionarse a las diferencias hormonales producidas durante la pubertad entre ambos sexos ${ }^{7}$. Finalmente, también se ha demostrado que los polimorfismos $-174 \mathrm{G}>\mathrm{C}$ y -597 G>A en el gen promotor de la IL-6 estarían asociados con hiperandrogenismo en mujeres sin diabetes mellitus y que la presencia del alelo $G$ se asociaría con elevados niveles de IL-6, cortisol basal, 11 deoxicortisol, 17 hidroxiprogesterona y testosterona ${ }^{17}$.

El propósito de este estudio fue describir la frecuencia alélica y genotípica del polimorfismo -174 G/C del gen IL-6 y analizar su posible asociación con índice de masa corporal (IMC) y control metabólico en mujeres con DM1.

\section{Pacientes y Metodología}

El estudio comprendió un total de 248 mujeres; 145 mujeres con DM1 y 103 mujeres controles. Las pacientes con DM1 fueron reclutadas y seleccionadas en el IDIMI y los criterios de inclusión fueron: insulinopenia, uso de insulina desde el momento del diagnóstico, duración de la diabetes mayor a dos años. Los criterios de exclusión fueron los siguientes: DM2 u otro tipo de diabetes, período de luna de miel; función de tiroides anormal; nivel elevado de creatinina, uso de píldoras anticonceptivas, esteroides o cualquier otro tipo de medicación y la presencia de otras patologías crónicas tales como: síndromes genéticos, enfermedad celíaca, enfermedad renal, enfermedad hepática, cardiaca o desnutrición. El grupo control incluyó a mujeres sanas que tenían niveles de glucosa nor- males, sin historia de hiperandrogenismo, ciclos menstruales regulares y sin historia de otras enfermedades. Este grupo estuvo formado por mujeres del personal del IDIMI, amigas no diabéticas de las pacientes y niñas seleccionadas al azar de colegios cercanos al IDIMI.

\section{Estudio clínico}

En todas las mujeres del estudio se evaluó:

a) Antropometría: El peso fue medido usando una escala Seca convencional con una precisión de $100 \mathrm{~g}$ y la talla fue medida con un stadiometro Harpenden. La antropometría en las niñas más jóvenes o mayores de 19 años se reporta como SDS de desviación estándar o como datos brutos, respectivamente. El score de desviación estándar (SDS) se calculó para la talla, peso e IMC, utilizando curvas estándar NCHS para las niñas menores de 19 años ${ }^{18}$.

b) Control metabólico: En las pacientes con DM1 se midió hemoglobina glicosilada (HbA1c) usando un sistema automático (DCA-2000, Bayer Diagnostics, Tarrytown, NY), cada 3 meses, también se registro el esquema de tratamiento con insulina y de las dosis de insulina usada los diez días previos al control, además se registró el promedio de la glicemia de ayuno de los últimos 10 días.

El estudio fue aprobado por el Comité de Ética del Hospital San Borja-Arriarán. Las mujeres adultas firmaron el consentimiento informado $y$, en el caso de las niñas o adolescentes, este fue firmado por sus padres. Se obtuvo también el asentimiento escrito de parte de las niñas y adolescentes.

\section{Análisis genético}

En todas las pacientes con DM1 y controles, se obtuvo ADN a partir de una extracción de sangre periférica $(3 \mathrm{ml})$ empleando técnicas de extracción estándar (Método de Chomczynski, Winkler, Santiago. Chile). El ADN fue cuantificado a través de espectrofotometría a 260/280 nm para ser utilizado en la amplificación específica de la región en estudio (gen promotor de IL-6; posición 939-1102, GenBank accesión nro. Y00081.1; -222 a -59 relativo al sitio de comienzo de la transcripción). Los partidores utilizados fueron $5^{\prime}$-GCC TCA ATG ACG ACC TAA GC-3’ y 5'-TCA TGG GAA AAT CCC ACA TT-3’. La reacción de amplificación se realizó en un volumen total de 20 $\mu \mathrm{L}$ con la siguiente mezcla: $50 \mu \mathrm{M}$ de dNTP (Life 
Technologies, Paisley, UK), $1,5 \mu \mathrm{M}$ de $\mathrm{MgCl}_{2}, 0.5 \mathrm{U}$ Taq-polimerasa (Life Technologies, Paisley, UK), 1 $\mu$ mol de cada partidor y 40 ng de ADN genómico. Las condiciones de reacción se estandarizaron como: un ciclo de 5 minutos a $95^{\circ} \mathrm{C}$ seguido por 37 ciclos de 30 s en $95^{\circ}, 30$ s a $58^{\circ} \mathrm{C}$ y 30 s a $72^{\circ} \mathrm{C}$ y finalmente un ciclo de 10 minutos a $72^{\circ} \mathrm{C}$. El producto amplificado fue digerido con $2 \mathrm{U}$ de la enzima NlaIII (New England Biolabs, Beverly, MA, USA) por $16 \mathrm{~h}$ a $37^{\circ} \mathrm{C}$. El genotipo homocigoto G/G estuvo constituído por un fragmento de 163 $\mathrm{pb}$, el genotipo $\mathrm{C} / \mathrm{C}$ por dos fragmentos de $111 \mathrm{pb}$ y $52 \mathrm{pb}$ y el genotipo heterocigoto formado por los fragmentos $163 \mathrm{pb}, 111 \mathrm{pb}$ y $52 \mathrm{pb}$ respectivamente. Los fragmentos digeridos fueron visualizados en geles de agarosa al 2,5\%.

\section{Analisis estadístico}

Las comparaciones de frecuencia alélicas y genotípicas entre casos y controles fueron analizadas mediante las pruebas de $\chi^{2}$ y prueba exacta de Fisher utilizando el programa SHESIS (URL: http://202.120.7.14/analysis/myAnalysis.php). Se evaluó la concordancia de las frecuencias genotípicas con respecto al equilibrio de Hardy-Weinberg mediante la prueba exacta de $\chi^{2}$. La comparación entre las características clínicas y antropométricas del grupo DM1 versus el grupo control se realizaron con prueba $t$ de Student. Se realizó análisis de regresión para evaluar la asociación entre la carga genotípica y las variables IMC, IMC-SDS, control metabólico e insulina. Los valores fueron considerados estadísticamente significativos cuando $p<0,05$. Los datos fueron analizados utilizando el paquete estadístico STATA 10 (Stata Statistical Software 1984-2009).

\section{Resultados}

Las características de ambos grupos de estudio se presentan en la Tabla 1. Los rangos de edad de ambos grupos fue semejante (2,8-42,7 en DM1 y 2,0-44,7 años en controles), y además las edades fueron semejantes en el grupo adolescente con DM1 comparado con el grupo control. En adultas las edades también fueron semejantes en ambos grupos. El IMC e IMC-Z-score, en $>$ de 19 años $y \leq 19$ años, respectivamente, fue semejante en DM1 versus controles. Tampoco se observaron diferencias en el control metabólico al comparar las mujeres adultas con DM1 versus las adolescentes con esta patología.

La Tabla 2 muestra el análisis de frecuencias genotípicas y alélicas para el polimorfismo -174 G/C de IL-6. La distribución porcentual del alelos mostró una mayor frecuencia del alelo $\mathrm{C}$ en los casos con DM1 al compararlos con las mujeres controles ( $21 \%$ versus $14,1 \%$; $p=0,047)$; no obstante no se observaron diferencias al comparar la frecuencia genotípica de este polimorfismo en pacientes con DM1 versus controles ( $\mathrm{p}$-value global de $\mathrm{p}=0,110$ ).

Finalmente, la Tabla 3 muestra el análisis de regresión entre el genotipo IL-6 y diversas variables antropométricas y metabólicas. En ella es posible observar una asociación positiva entre el genotipo

Tabla 1. Características clínicas y antropométricas de ambos grupos

\begin{tabular}{|lcc|}
\hline & Grupo DM1 (n = 145) & Grupo control (n = 103) \\
Niñas/adolescentes (<19 años) & 101 & 54 \\
N & $12,1 \pm 4,3$ & $12,9 \pm 3,8$ \\
Edad (años) & $0,85 \pm 0,69$ & $0,57 \pm 1,5$ \\
IMC-SDS & $8,9 \pm 1,7$ & - \\
HbA1c (\%) & & \\
Mujeres adultas ( $\geq 19$ años) & 44 & 49 \\
N & $29,6 \pm 8,4$ & $30,5 \pm 7,7$ \\
Edad (años) & $24,2 \pm 2,8$ & $24,9 \pm 3,5$ \\
IMC (kg/m $\left.{ }^{2}\right)$ & $8,4 \pm 2,2$ & - \\
HbA1c (\%) & & \\
\hline
\end{tabular}


Tabla 2. Distribución de frecuencias genotípicas y alélicas para el polimorfismo -174 G/C del gen IL-6 en pacientes con DM1 y controles

\begin{tabular}{|c|c|c|c|c|c|}
\hline \multirow[t]{2}{*}{ Variante genética } & \multicolumn{2}{|c|}{ DM1 $(n=145)$} & \multicolumn{2}{|c|}{ Controles $(n=103)$} & \multirow[t]{2}{*}{ p-value } \\
\hline & $\mathbf{n}$ & Frec. & $\mathbf{n}$ & Frec. & \\
\hline$C / C$ & 6 & 0,041 & 1 & 0,010 & 0,110 \\
\hline$C / G$ & 49 & 0,338 & 27 & 0,262 & \\
\hline $\mathrm{G} / \mathrm{G}$ & 90 & 0,621 & 75 & 0,728 & \\
\hline$\%$ allele C & 21,0 & & 14,1 & & 0,047 \\
\hline$\%$ allele G & 79,0 & & 85,9 & & \\
\hline Hardy-Weinberg $p$-value & 0,83 & & 0,39 & & \\
\hline \multicolumn{6}{|l|}{ Modelo Recesivo } \\
\hline $\mathrm{CC}+\mathrm{CG}$ & 55 & 0,379 & 28 & 0,272 & ns \\
\hline GG & 90 & 0,621 & 75 & 0,728 & \\
\hline \multicolumn{6}{|l|}{ Modelo Dominante } \\
\hline $\mathrm{GG}+\mathrm{CG}$ & 139 & 0,959 & 102 & 0,990 & ns \\
\hline CC & 6 & 0,041 & 1 & 0,010 & \\
\hline
\end{tabular}

Tabla 3. Análisis de regresión logística para el efecto del polimorfismo -174 G/C (carga genotípica G/G) sobre el IMC, edad de diagnóstico y control metabólico en pacientes con DM1

\begin{tabular}{|lcc|}
\hline & Coeficiente $-\beta$ & p-value \\
\hline Genotipo IL-6 sobre IMC en DM1 > 19 años & 1,55 & 0,170 \\
Genotipo IL-6 sobre IMC en Controles > 19 años & 1,45 & 0,086 \\
Genotipo IL-6 sobre IMC-SDS en DM1 < 19 años & 0,13 & 0,234 \\
Genotipo IL-6 sobre IMC-SDS en Controles < 19 años & 0,31 & 0,331 \\
Genotipo IL-6 sobre la edad de diagnóstico en DM1 & 3,12 & 0,014 \\
Genotipo IL-6 sobre dosis de insulina en DM1 & $-0,04$ & 0,946 \\
Genotipo IL-6 sobre control metabólico en DM1 & $-0,10$ & 0,706 \\
\hline
\end{tabular}

El IMC fue analizado como puntaje de desviación estándar (SDS) en mujeres menores de 19 años.

-174 G/C del gen de IL-6 y edad de diagnóstico de la enfermedad ( $\mathrm{p}=0,014)$, lo que significa que el genotipo GG se asocia a mayor edad del diagnóstico. En relación al IMC sólo se observó una leve tendencia entre genotipo -174 G/C del gen IL-6 y el IMC en mujeres controles sanas mayores de 19 años $(\mathrm{p}=0,086)$.

\section{Discusión}

La IL-6 es una citoquina proinflamatoria, cuyos niveles elevados se relacionan con estados de insulino-resistencia ${ }^{19}$. Para el gen de IL-6, se han descrito diferentes polimorfismos en su región promotora que afectan la transcripción del gen y con ello los niveles circulantes de esta citoquina ${ }^{13}$. Diversos estudios han descrito una posible asociación de uno de sus polimorfismos $(-174 \mathrm{G} / \mathrm{C})$ con susceptibilidad a la DM1, específicamente con la presencia del genotipo $\mathrm{G} / \mathrm{G}^{15}$.

Las observaciones de este estudio demostraron una alta frecuencia de este genotipo en la población chilena, tanto en pacientes con DM1, como en controles. Estos resultados difieren de lo descrito en población europea donde el genotipo más frecuente corresponde a la combinación 
heterocigota G/C. La mayoría de los estudios poblacionales realizados en Europa muestran a este genotipo como el más prevalente y al genotipo $\mathrm{C} / \mathrm{C}$ como el de menor frecuencia. En nuestro caso, existe concordancia para la baja frecuencia del genotipo $\mathrm{C} / \mathrm{C}^{13}$.

En otras patologías de carácter autoinmune como la artritis reumatoidea, se ha descrito que el genotipo C/C se correspondería con una forma de protección ya que este genotipo impacta en una menor expresión del gen y en bajos niveles circulantes de IL- 6 circulante, lo que podría constituir un beneficio para dichos pacientes ${ }^{20}$. Otras patologías con participación del componente inmunológico en la DM1, como la enfermedad peridontal ${ }^{20}$ y la enfermedad celíaca ${ }^{21}$, muestran que la presencia del alelo G estaría asociado a un mayor riesgo.

Nuestro estudio describe una muy baja frecuencia del genotipo $\mathrm{C} / \mathrm{C}$, fenómeno que también ha sido descrito en otras poblaciones del sur de China y en coreanos, donde la frecuencia de esta combinación es muy baja o nula ${ }^{22,23}$.

La frecuencia alélica observada por este estudio mostró que el alelo $\mathrm{G}$ es el de mayor prevalencia, tanto en el grupo de pacientes con DM1 (79\%), como en el grupo control (86\%). Hay diversos estudios que han relacionado la presencia del alelo $\mathrm{G}$ con una mayor transcripción del gen $\mathrm{y}$ por ende con una mayor producción de IL- $6^{14}$. Kristiansen y $\operatorname{cols}^{18}$ demostraron que el alelo G se asocia con DM1, indicando que la presencia del alelo C, que es menos activo en ausencia de estrógenos, se tornaría más activo en ausencia de este esteroide lo cual podría relacionarse con un inicio más temprano de la DM1. Hermann y $\operatorname{cols}^{24}$ describen una asociación similar con un efecto de epistásis de este polimorfismo junto al gen del factor de necrosis tumoral alfa (TNF- $\alpha$ ) sobre la edad de inicio de la DM1. Nuestros datos serían compatibles con esta hipótesis, dado que las pacientes con DM1 portadoras del genotipo G/G tienen una edad de debut más tardía de la enfermedad en comparación con las mujeres con otros genotipos (coeficiente $\beta=3,12 ; p=0,014$ ).

La asociación de la IL-6 en la homeostasis energética está ampliamente documentada ${ }^{13}$, de forma que su posible relación con el desarrollo de obesidad podría estar mediada por las acciones de esta citoquina, tal vez en función del polimorfismo $-174 \mathrm{G} / \mathrm{C}$. Existen varios reportes en la literatura que han relacionado la presencia del genotipo $\mathrm{G} / \mathrm{G}$ con insulino-resistencia y dislipidemia ${ }^{25}$ y con incrementos del IMC y leptina ${ }^{26,27}$. Estudios preliminares realizados en el año 2001, ya planteaban la posibilidad de una posible asociación con enfermedad cardiovascular e hipertensión arterial ${ }^{28}$, proponiendo que esta asociación involucraba al componente inflamatorio. Este antecedente fue abordado en el año 2006, donde se reporta la asociación de este polimorfismo y en especial del genotipo G/G con altas concentraciones de proteína C reactiva (usPCR) en individuos obesos sometidos a reducción de peso ${ }^{29}$. Sin embargo, esta asociación no ha podido ser confirmada por estudios familiares donde es el genotipo $\mathrm{C} / \mathrm{C}$ el asociado con altos niveles de usPCR ${ }^{30}$. Recientemente, se ha descrito una fuerte asociación del alelo $\mathrm{C}$ con bajos niveles de glucosa, insulina y HOMA en mujeres con síndrome de ovario poliquístico ${ }^{31}$ indicando que ser portador del genotipo G/G se asocia con las anormalidades metabólicas de este síndrome.

En este sentido, nuestro estudio sólo encontró una mayor tendencia de asociación para la presencia del alelo G con mayor IMC en mujeres adultas con DM1 $(\mathrm{p}=0,086)$, no así en adolescentes con DM1, ni en el grupo control.

Se ha planteado que el polimorfismo - 174 G/C del gen IL-6 podría estar asociado con un incremento del IMC en pacientes con DM1 desde donde ejercería algún papel en los mecanismos de inflamación descritos en pacientes con DM1. Sin embargo, nuestros resultados no encontraron ninguna asociación significativa con IMC o SDSIMC indicando que dicho polimorfismo no sería relevante, al menos en este grupo de estudio con respecto a la ganancia de peso.

Agradecimientos: Este trabajo fue financiado a través del Proyecto FONDECYT 1050452 (Dra. Ethel Codner). Los autores agradecen a la Sra. Alejandra Ávila el excelente apoyo de enfermería, $\mathrm{y}$ a todas las pacientes $\mathrm{y}$ controles que participaron de este estudio y el apoyo logístico aportado por el Hospital San Borja Arriarán.

\section{Referencias}

1. Dabelea D. The accelerating epidemic of childhood diabetes. The Lancet 2009; 373: 1999-2000. 
2. Mehers KL, Gillespie KM The genetic basis for type 1 diabetes. Br Med Bull 2008; 88: 115-29.

3. Merriman TR.Type 1 diabetes, the A1 milk hypothesis and vitamin D deficiency. Diabetes Res Clin Pract 2009; 83: 149-56.

4. TEDDY Study Group, The environmental determinants of diabetes in the young (TEDDY) study, Ann NY Acad Sci 2008; 1150: 1-13.

5. Pang TT, Narendran P. Addressing insulin resistance in Type 1 diabetes. Diab Med 2008; 25: 1015-24.

6. Eizirik DL, Colli ML, Ortis F. The role of inflammation in insulitis and beta-cell loss in type 1 diabetes. Nat Rev Endocrinol 2009; 5: 219-26.

7. Codner E. Estrogen and type 1 diabetes mellitus. Pediatr Endocrinol Rev 2008; 6: 228-34.

8.- Ishihara K, Hirano T. IL-6 in autoimmune disease and chronic inflammatory proliferative disease. Cytokine Growth Factor Rev 2002; 13: 357-68.

9. Vozarova B, Weyer C, Hanson K, Tatarani P, Bogardus C, Pratley RE. Circulating interleukin-6 in relation to adiposity, insulin actino, and insulin secretion. Obes Res 2001; 9: 414-7.

10. Kern Pa, Ranganathan S, Li C, Wood L, Ranganathan G. Adipose tissue tumor necrosis facor and IL-6 expression in human obesity and insulin resistance. Am J Physiol Endocrinol Metab 2001; 280: E745-E751.

11. Bastard JP, Jardel C, Bruckert E, Blondy P, Capeau J, Laville M, et al. Elevated levels of interleukin- 6 are reduced in serum and subcutaneous adipose tissue of obese women after weight loss. J Clin Endocrinol Metab 2000; 85: 3338-42.

12. Pradhan AD, Manson JE, Rifai N, Buring JE, Ridker PM. $C$ reactive protein, interleukin 6 , and risk of developing type 2 diabetes mellitus. JAMA 2001; 286: 327-34.

13. Qi L, Zhang C, van Dam RM, Hu FB. Interleukin-6 genetic variability and adiposity: associations in two prospective cohorts and systematic review in 26,944 individuals. J Clin Endocrinol Metab 2007; 92: 3618-25.

14. Fishman D, Faulds G, Jeffery R, Mohamed-Ali V, Yudkin JS, Humphries S, Woo P. The effect of novel polymorphisms in the interleukin-6 (IL-6) gene on IL-6 transcription and plasma IL-6 levels, and an association with systemic-onset juvenile chronic arthritis. J Clin Invest 1998, 102: 1369-76.

15. Jahromi MM, Millward BA, Demaine AG.A polymorphism in the promoter region of the gene for interleukin- 6 is associated with susceptibility to type 1 diabetes mellitus. J Interferon Cytokine Res 2000; 20: 885-8.

16. Gillespie KM, Nolsoe R, Betin V, Kristiansen OP, Bingley PJ, Mandrup-Poulsen T, et al. Is Puberty an accelerator of type 1 Diabetes in IL6-174CC Females? Diabetes
2005; 54: 1245-8.

17. Kristiansen OP, Nolsoe RL, Larsen L, Gjesing AM, Johannesen J, Larsen ZM, $t$ al. Association of a functional $17 \beta$-estradiol sensitive IL6-174G/C promoter polymorphism whit early-onset type 1 diabetes in females. Human Molecular Genetics 2003; 12: 1101-10.

18. Ogden CL, Kuczmarski JR, Flegal KM, Mei Z, Guo S, Wei R, et al. Centers for Disease Control and Prevention 2000 growth charts for the United States: improvements to the 1977 National Center for Health Statistics version. Pediatrics 2002; 109: 45-60.

19. Kristiansen OP, Mandrup-Poulsen T. Interleukin-6 and diabetes: the good, the bad, or the indifferent?. Diabetes 2005; 54: S114-24.

20. Raunio T, Knuuttila M, Hiltunen L, Karttunen R, Vainio O, Tervonen T. IL-6(-174) genotype associated with the extent of periodontal disease in type 1 diabetic subjects. J Clin Periodontol 2009; 36: 11-7.

21. Myśliwiec M, Myśliwska J, Zorena K, Balcerska A, Malinowska E, Wiśniewski P.Interleukin $6-174(\mathrm{G}>\mathrm{C})$ gene polymorphism is related to celiac disease and autoimmune thyroiditis coincidence in diabetes type 1 children. Diabetes Res Clin Pract 2008; 82: 108-12.

22. Lim CS, Zheng S, Kim YS, Ahn C, Han JS, Kim S, et al. The $-174 \mathrm{G}$ to $\mathrm{C}$ polymorphism of interleukin- 6 gene is very rare in koreans. Cytokine 2002; 19: 52-4.

23. Zhai R, Liu G, Yang C, Huang C, Wu C, Christiani DC. The $\mathrm{G}$ to $\mathrm{C}$ polymorphism at -174 of the interleukin- 6 gene is rare in a Southern Chinese population. Pharmacogenetics 2001; 8: 699-701.

24. Hermann C, Krikovszky D, Füst G, Kovács M, Körner A, Szabó A, et al. Association between interleukin-6 polymorphism and age-at-onset of type 1 diabetes. Epistatic influences of the tumor necrosis factor-alpha and interleukin-1 beta polymorphisms. Eur Cytokine Netw 2005; 16: 277-81.

25. Hamid YH, Rose CS, Urhammer SA, Glümer C, Nolsøe $\mathrm{R}$, Kristiansen OP, et al. Variations of the interleukin-6 promoter are associated with features of the metabolic syndrome in Caucasian Danes. Diabetologia 2005; 48: 251-60.

26. Wernstedt I, Eriksson AL, Berndtsson A, Hoffstedt J, Skrtic S, Hedner T, et al. A common polymorphism in the interleukin- 6 gene promoter is associated with overweight. Int J Obes Relat Metab Disord 2004; 28: 1272-9.

27. Di Renzo L, Bertoli A, Bigioni M, Del Gobbo V, Premrov MG, Calabrese V, et al. Body composition and -174G/C interleukin-6 promoter gene polymorphism: association with progression of insulin resistance in normal weight obese syndrome. Curr Pharm Des 2008; 14: 2699-706. 
28. Humphries SE, Luong LA, Ogg MS, Hawe E, Miller GJ. The interleukin-6 -174 G/C promoter polymorphism is associated with risk of coronary heart disease and systolic blood pressure in healthy men. Eur Heart J 2001; 22: 2243-52.

29. Eklund C, Nenonen A, Kukkonen-Harjula K, Borg P, Fogelholm M, Laine S, et al. Association of the IL6174(G/C) polymorphism with C-reactive protein concentration after weight loss in obese men. Eur Cytokine Netw 2006; 17: 131-5.
30. Guzmán-Guzmán IP, Muñoz-Valle JF, Flores-Alfaro E, Salgado-Goytia L, Salgado-Bernabé AB, Parra-Rojas I. Interleukin- 6 gene promoter polymorphisms and cardiovascular risk factors. A family study. Dis Markers 2010; 28: 29-36.

31. Vural P, Değirmencioğlu S, Saral NY, Akgül C.Tumor necrosis factor alpha (-308), interleukin-6 (-174) and interleukin-10 (-1082) gene polymorphisms in polycystic ovary syndrome. Eur J Obstet Gynecol Reprod Biol 2010; 150: 61-5. 